Article

\title{
Sharp Bounds on the Higher Order Schwarzian Derivatives for Janowski Classes
}

\author{
Nak Eun Cho ${ }^{1}$, Virendra Kumar ${ }^{2, *}$ and V. Ravichandran ${ }^{3}$ \\ 1 Department of Applied Mathematics, Pukyong National University, Busan 48513, Korea; necho@pknu.ac.kr \\ 2 Department of Mathematics, Ramanujan college, University of Delhi, H-Block, Kalkaji, \\ New Delhi 110019, India \\ 3 Department of Mathematics, National Institute of Technology, Tiruchirappalli, Tamil Nadu 620015, India; \\ ravic@nitt.edu; vravi68@gmail.com \\ * Correspondence: vktmaths@yahoo.in
}

Received: 25 July 2018; Accepted: 14 August 2018; Published: 18 August 2018

\begin{abstract}
Higher order Schwarzian derivatives for normalized univalent functions were first considered by Schippers, and those of convex functions were considered by Dorff and Szynal. In the present investigation, higher order Schwarzian derivatives for the Janowski star-like and convex functions are considered, and sharp bounds for the first three consecutive derivatives are investigated. The results obtained in this paper generalize several existing results in this direction.
\end{abstract}

Keywords: higher order Schwarzian derivatives; Janowski star-like function; Janowski convex function; bound on derivatives

MSC: 30C45; 30C50; 30C80

\section{Introduction}

Let $\mathcal{A}$ be the class of analytic functions defined on the unit disk $\mathbb{D}:=\{z \in \mathbb{C}:|z|<1\}$ and having the form:

$$
f(z)=z+a_{2} z^{2}+a_{3} z^{3}+\cdots .
$$

The subclass of $\mathcal{A}$ consisting of univalent functions is denoted by $\mathcal{S}$. An analytic function $f$ is subordinate to another analytic function $g$ if there is an analytic function $w$ with $|w(z)| \leq|z|$ and $w(0)=0$ such that $f(z)=g(w(z))$, and we write $f \prec g$. If $g$ is univalent, then $f \prec g$ if and only if $f(0)=g(0)$ and $f(\mathbb{D}) \subseteq g(\mathbb{D})$. The classes $\mathcal{S}^{*}$ and $\mathcal{K}$ of star-like and convex functions, respectively, are among the most studied subclasses of $\mathcal{S}$. These classes are defined, respectively, as:

$$
\mathcal{S}^{*}:=\left\{f \in \mathcal{S}: \operatorname{Re}\left(\frac{z f^{\prime}(z)}{f(z)}\right)>0, z \in \mathbb{D}\right\}
$$

and:

$$
\mathcal{K}:=\left\{f \in \mathcal{S}: \operatorname{Re}\left(1+\frac{z f^{\prime \prime}(z)}{f^{\prime}(z)}\right)>0, z \in \mathbb{D}\right\} .
$$

The Koebe function $k(z)=z /(1-z)^{2} \in \mathcal{S}^{*}$ and $z /(1-z) \in \mathcal{K}$.

General forms of these classes were considered by Janowski [1]. For $-1 \leq B<A \leq 1$, these classes are defined by:

$$
\mathcal{S}^{*}[A, B]:=\left\{f \in \mathcal{S}: \frac{z f^{\prime}(z)}{f(z)} \prec \frac{1+A z}{1+B z}\right\} \text { and } \mathcal{K}[A, B]:=\left\{f \in \mathcal{S}: 1+\frac{z f^{\prime \prime}(z)}{f^{\prime}(z)} \prec \frac{1+A z}{1+B z}\right\} .
$$


These classes are called the class of Janowski star-like and Janowski convex functions, respectively. On specializing the parameters $A$ and $B$, we get several well-known classes such as $\mathcal{S}^{*}:=\mathcal{S}^{*}[1,-1]$ and $\mathcal{K}:=\mathcal{K}[1,-1]$. The functions $h_{0}$ and $k_{0}$ defined by:

$$
h_{0}(z)= \begin{cases}z(1+B z)^{\frac{A}{B}-1}, & B \neq 0 \\ z e^{A z,} & B=0\end{cases}
$$

and:

$$
k_{0}(z)= \begin{cases}\frac{1}{A}\left[(1+B z)^{\frac{A}{B}}-1\right], & B \neq 0, A \neq 0 ; \\ \frac{1}{B} \log (1+B z), & A=0 ; \\ \frac{1}{A}\left[e^{A z}-1\right], & B=0 .\end{cases}
$$

belong to the classes $\mathcal{S}^{*}[A, B]$ and $\mathcal{K}[A, B](-1 \leq B<A \leq 1)$, respectively. In particular, $2 z /(2-z) \in$ $\mathcal{S}^{*}[1 / 2,-1 / 2]$ and $4 z /(2-z)^{2} \in \mathcal{K}[1 / 2,-1 / 2]$.

The quantity $a_{2}^{2}-a_{3}$ is associated with the Schwarzian derivative of function $f \in \mathcal{S}$. Recall that the Schwarzian derivative of a locally univalent function $f$ is defined by:

$$
\mathbf{S}(f)(z):=\left(\frac{f^{\prime \prime}(z)}{f^{\prime}(z)}\right)^{\prime}-\frac{1}{2}\left(\frac{f^{\prime \prime}(z)}{f^{\prime}(z)}\right)^{2}
$$

which is an important quantity in univalent function theory. For example, the quantity $a_{3}-\mu a_{2}^{2}=$ $\left(f^{\prime \prime \prime}(0)-3 \mu\left(f^{\prime \prime}(0)\right)^{2} / 2\right) / 6$ is called the Fekete-Szegö functional, and finding the sharp bound on modulus of this quantity is popularly known as the Fekete-Szegö problem. Nehari [2] (see also [3]) proved that the necessary condition for an analytic function $f$ to be in the class $\mathcal{S}$ is $|\mathbf{S}(f)(z)| \leq$ $6\left(1-|z|^{2}\right)^{-2}$, and the sufficient condition is $|\mathbf{S}(f)(z)| \leq 2\left(1-|z|^{2}\right)^{-2}$. In both directions, the results are the best possible in the sense that the constants two and six cannot be replaced by the smaller numbers. The sharpness of the later condition was verified by Hille [4]. The first inequality is sharp in the case of the Koebe function, whereas the sharpness in the second can be seen in the case of the function $f_{0}(z)=(1 / 2) \log ((1+z) /(1-z))$. Later, Nehari [5] proved that if $f$ is a convex function, then $|\mathbf{S}(f)(z)| \leq 2\left(1-|z|^{2}\right)^{-2}$.

Aharanov and Harmelin [6] studied the higher order Schwarzian derivatives $\sigma_{n}(f)$ with invariance under composition on the left by Möbius transformations $T, \sigma_{n}(T \circ f)=\sigma_{n}(f)$, and their relation to univalence of the function $f$. The higher order Schwarzian derivative is defined as follows (see $[6,7])$ :

$$
\sigma_{3}(f)=\mathbf{S}(f)
$$

and for any integer $n \geq 4$, it is given by:

$$
\sigma_{n+1}(f)=\left(\sigma_{n}(f)\right)^{\prime}-(n-1) \sigma_{n}(f) \frac{f^{\prime \prime}}{f^{\prime}} .
$$

In particular,

$$
\sigma_{4}(f)=\frac{f^{(4)}}{f^{\prime}}-6 \frac{f^{\prime \prime \prime} f^{\prime}}{f^{\prime 2}}+\left(\frac{f^{\prime \prime}}{f^{\prime}}\right)^{3}
$$

and for:

$$
\sigma_{5}(f)=\frac{f^{(5)}}{f^{\prime}}-10 \frac{f^{(4)} f^{\prime \prime}}{f^{\prime 2}}-6\left(\frac{f^{\prime \prime \prime}}{f^{\prime}}\right)^{2}+48 \frac{f^{\prime \prime \prime} f^{\prime 2}}{f^{\prime 3}}-36\left(\frac{f^{\prime \prime}}{f^{\prime}}\right)^{4} .
$$

Schippers [7] derived the differential equation for the Loewner flow of the Schwarzian derivative of univalent functions and used this to investigate the bounds on the modulus of higher order Schwarzian derivatives. These bounds were shown to be sharp in the case of the Koebe function. He also proved certain two-point distortion theorems for the higher order Schwarzian derivatives in terms of the hyperbolic metric. Later, the higher order Schwarzian derivatives for convex functions 
were considered by Dorff and Szynal [8]. Since the class $\mathcal{K}$ is linearly invariant (see [9]), so there is no loss in restricting consideration to $\sigma_{n}(f)(0)=: \mathbf{S}_{n}$. From the above definition of $\sigma_{n}(f)$, we see that $\mathbf{S}_{3}=\sigma_{3}(f)(0)=6\left(a_{3}-a_{2}^{2}\right), \mathbf{S}_{4}=\sigma_{4}(f)(0)=24\left(a_{4}-3 a_{2} a_{3}+2 a_{2}^{3}\right)$ and $\mathbf{S}_{5}=\sigma_{5}(f)(0)=$ $24\left(5 a_{5}-20 a_{2} a_{4}-9 a_{3}^{2}+48 a_{3} a_{2}^{2}-24 a_{2}^{4}\right)$. Droff and Szynal proved that $\left|\mathbf{S}_{3}\right| \leq 2,\left|\mathbf{S}_{4}\right| \leq 4$ and $\left|\mathbf{S}_{5}\right| \leq 12$ with inequality in the case of the function:

$$
f_{n}(z)=\int_{0}^{z}\left(1-t^{n-1}\right)^{-\frac{2}{n-1}} d t, n=3,4,5 .
$$

They also conjectured that the maximal value of $\left|\mathbf{S}_{n}\right|$ for $n=6,7,8, \cdots$ is attained in the case of the function $f_{n}$ defined above.

In general, it is not so easy for researchers to deal with the higher order Schwarzian derivatives as the methods in geometric function theory known at present time are not substantial enough. However, they have a very important role in geometric/univalent function theory. In particular, Gal [10], by using the powerful method of admissible functions of Miller and Mocanu [11], investigated the geometric criterion of univalence, which combines higher order Schwarzian derivatives with those of the Ruscheweyh and Sălăgean operators. Therefore, it is very natural to consider higher order Schwarzian derivatives for various geometric results of analytic functions. In this direction, Tamanoi [12], investigated various properties of higher Schwarzian derivatives and their relation with combinatorial polynomials. Tamanoi also proved that the higher Schwarzian derivatives are Möbius invariant; see [12] (p. 135 Theorems 3-3(ii)). Later, in 2011, Kim and Sugawa [13] investigated relations between the Aharonov invariants $([13,14])$ and Tamanoi's Schwarzian derivatives of higher order and gave a recursive formula for Tamanoi's Schwarzians. In the same paper, they proposed a new definition of invariant Schwarzian derivatives of a non-constant holomorphic function between Riemann surfaces with conformal metrics. In 2011, Kim and Sugawa reviewed the Peschl-Minda derivatives [15,16] and Schwarzian derivatives of higher order due to Aharonov [14], Tamanoi [12] and Kim and Sugawa [13] for a non-constant holomorphic map between Riemann surfaces with conformal metrics. They also proved that the higher-order Schwarzian derivatives of Aharonov and Tamanoi cannot be extended to holomorphic functions between projective Riemann surfaces unlike the classical Schwarzian derivatives. The higher order Schwarzian derivative are useful in the study of the properties of non-linear dynamical system and has been studied extensively by several researchers [17]. For many applications of the higher order Schwarzian derivatives related to the real functions, the reader may refer to $[17,18]$ and the references cited therein. Kwon and Sim [19], in 2017, using the theory of admissible functions investigated some sufficient conditions for normalized analytic functions to be star-like, associated with Tamanoi's Schwarzian derivative of third order.

Motivated by the works of Schippers [7] and Dorff and Szynal [8] and other related works cited above, in this paper, we shall consider the higher order Schwarzian derivatives for Janowski star-like and convex functions. The sharp bound on the first three consecutive Schwarzian derivatives for Janowski star-like and convex functions is investigated. We shall also point out some relevant connections of our results with the existing result. Several examples in support of our main results are also given with explanations. To prove our results, we need the following results:

Let $\mathcal{B}$ be the class of Schwarz functions consisting of analytic functions of the form $w(z)=$ $c_{1} z+c_{2} z^{2}+c_{3} z^{3}+\cdots(z \in \mathbb{D})$ and satisfying the condition $|w(z)|<1$ for $z \in \mathbb{D}$. Let $\mathcal{P}$ denote the class of analytic functions of the form $p(z)=1+p_{1} z+p_{2} z^{2}+p_{3} z^{3}+\cdots$ for which $\operatorname{Re} p(z)>0(z \in \mathbb{D})$. The following correspondence between the classes $\mathcal{B}$ and $\mathcal{P}$ holds:

$$
p \in \mathcal{P} \text { if and only if } w(z)=\frac{p(z)-1}{p(z)+1} \in \mathcal{B}
$$


Comparing coefficients in (4), we have:

$$
c_{1}=\frac{p_{1}}{2}, c_{2}=\frac{2 p_{2}-p_{1}^{2}}{4}, c_{3}=\frac{4 p_{3}-4 p_{1} p_{2}+p_{1}^{3}}{8}, c_{4}=\frac{8 p_{4}-8 p_{1} p_{3}-4 p_{2}^{2}+6 p_{1}^{2} p_{2}-p_{1}^{4}}{16} .
$$

Consider the functional $\Psi(\mu, v)=\left|c_{3}+\mu c_{1} c_{2}+v c_{1}^{3}\right|$ for $w \in \mathcal{B}$ and $\mu, v \in \mathbb{R}$.

Lemma 1. If $w \in \mathcal{B}[20]$ (p. 128 Lemma 2), then for any real numbers $\mu$ and $v$, we have:

$$
|\Psi(\mu, v)| \leq \begin{cases}1, & (\mu, v) \in \Omega_{1} \cup \Omega_{2} \cup\{(2,1)\} ; \\ |v|, & (\mu, v) \in \bigcup_{k=3}^{7} \Omega_{k} \\ \frac{2}{3}(|\mu|+1)\left(\frac{|\mu|+1}{3(|\mu|+v+1)}\right)^{1 / 2}, & (\mu, v) \in \Omega_{8} \cup \Omega_{9} ; \\ \frac{1}{3} v\left(\frac{\mu^{2}-4}{\mu^{2}-4 v}\right)\left(\frac{\mu^{2}-4}{3(v-1)}\right)^{1 / 2}, & (\mu, v) \in \Omega_{10} \cup \Omega_{11}-\{(2,1)\} ; \\ \frac{2}{3}(|\mu|-1)\left(\frac{|\mu|-1}{3(|\mu|-v-1)}\right)^{1 / 2}, & (\mu, v) \in \Omega_{12} .\end{cases}
$$

Here, the symbols $\Omega_{k}$ 's are defined as follows:

$$
\begin{gathered}
\Omega_{1}:=\left\{(\mu, v) \in \mathbb{R}^{2}:|\mu| \leq 1 / 2,|v| \leq 1\right\}, \\
\Omega_{2}:=\left\{(\mu, v) \in \mathbb{R}^{2}: \frac{1}{2} \leq|\mu| \leq 2, \quad \frac{4}{27}(|\mu|+1)^{3}-(|\mu|+1) \leq v \leq 1\right\}, \\
\Omega_{3}:=\left\{(\mu, v) \in \mathbb{R}^{2}:|\mu| \leq \frac{1}{2}, v \leq-1\right\}, \Omega_{4}:=\left\{(\mu, v) \in \mathbb{R}^{2}:|\mu| \geq 1 / 2, \quad v \leq-\frac{2}{3}(|\mu|+1)\right\}, \\
\Omega_{5}:=\left\{(\mu, v) \in \mathbb{R}^{2}:|\mu| \leq 2, v \geq 1\right\}, \Omega_{6}:=\left\{(\mu, v) \in \mathbb{R}^{2}: 2 \leq|\mu| \leq 4, v \geq \frac{1}{12}\left(\mu^{2}+8\right)\right\}, \\
\Omega_{8}:=\left\{(\mu, v) \in \mathbb{R}^{2}: \frac{1}{2} \leq|\mu| \leq 2,-\frac{2}{3}(|\mu|+1) \leq v \leq \frac{4}{27}(|\mu|+1)^{3}-(|\mu|+1)\right\}, \\
\Omega_{9}:=\left\{(\mu, v) \in \mathbb{R}^{2}:|\mu| \geq 2,-\frac{2}{3}(|\mu|+1) \leq v \leq \frac{2|\mu|(|\mu|+1)}{\mu^{2}+2|\mu|+4}\right\}, \\
\Omega_{10}:=\left\{(\mu, v) \in \mathbb{R}^{2}: 2 \leq|\mu| \leq 4, \frac{2|\mu|(|\mu|+1)}{\mu^{2}+2|\mu|+4} \leq v \leq \frac{1}{12}\left(\mu^{2}+8\right)\right\}, \\
\Omega_{11}:=\left\{(\mu, v) \in \mathbb{R}^{2}:|\mu| \geq 4, \frac{2|\mu|(|\mu|+1)}{\mu^{2}+2|\mu|+4} \leq v \leq \frac{2|\mu|(|\mu|-1)}{\mu^{2}-2|\mu|+4}\right\} \\
\Omega_{12}:=\left\{(\mu, v) \in \mathbb{R}^{2}:|\mu| \geq 4, \frac{2|\mu|(|\mu|-1)}{\mu^{2}-2|\mu|+4} \leq v \leq \frac{2}{3}(|\mu|-1)\right\} .
\end{gathered}
$$

The extremal functions, up to rotations, are of the form:

$$
w_{1}(z)=z^{3}, w_{2}(z)=z, w_{3}(z)=\frac{z\left(t_{1}-z\right)}{1-t_{1} z}, w_{4}(z)=\frac{z\left(t_{2}+z\right)}{1+t_{2} z}
$$

and $w_{5}(z)=c_{1} z+c_{2} z^{2}+c_{3} z^{3}+\cdots$, where the parameters $t_{1}, t_{2}$ and the coefficients $c_{i}$ are given by:

$$
t_{1}=\left(\frac{|\mu|+1}{3(|\mu|+v+1)}\right)^{1 / 2}, t_{2}=\left(\frac{|\mu|-1}{3(|\mu|-v-1)}\right)^{1 / 2}, c_{1}=\left(\frac{2 v\left(\mu^{2}+2\right)-3 \mu^{2}}{3(v-1)\left(\mu^{2}-4 v\right)}\right)^{1 / 2},
$$




$$
c_{2}=\left(1-c_{1}^{2}\right) e^{i \theta_{0}}, \quad c_{3}=-c_{1} c_{2} e^{i \theta_{0}}, \theta_{0}= \pm \arccos \left[\frac{\mu}{2}\left(\frac{v\left(\mu^{2}+8\right)-2\left(\mu^{2}+2\right)}{2 v\left(\mu^{2}+2\right)-3 \mu^{2}}\right)^{1 / 2}\right] .
$$

Lemma 2. If $w \in \mathcal{B}$ [21](see also, [22]), then for any complex numbers $\tau$, we have:

$$
\left|c_{2}-\tau c_{1}^{2}\right| \leq \max \{1 ;|\tau|\} .
$$

The result is sharp for the functions $w(z)=z$ or $w(z)=z^{2}$.

Lemma 3. Let $\hat{\alpha}, \hat{\beta}, \hat{\gamma}$ and $\hat{a}$ satisfy the inequalities [23] (p. 506 Lemma 2.1), $0<\hat{\alpha}<1,0<\hat{a}<1$ and:

$$
\begin{gathered}
8 \hat{a}(1-\hat{a})\left[(\hat{\alpha} \hat{\beta}-2 \hat{\gamma})^{2}+(\hat{\alpha}(\hat{a}+\hat{\alpha})-\hat{\beta})^{2}\right]+\hat{\alpha}(1-\hat{\alpha})(\hat{\beta}-2 \hat{a} \hat{\alpha})^{2} \leq 4 \hat{a} \hat{\alpha}^{2}(1-\hat{\alpha})^{2}(1-\hat{a}) . \\
\text { If } p(z)=1+p_{1} z+p_{2} z^{2}+p_{3} z^{3}+\cdots \in \mathcal{P} \text {, then: } \\
\left|\hat{\gamma} p_{1}^{4}+\hat{a} p_{2}^{2}+2 \hat{\alpha} p_{1} p_{3}-(3 / 2) \hat{\beta} p_{1}^{2} p_{2}-p_{4}\right| \leq 2 .
\end{gathered}
$$

\section{Main Results}

The following theorem gives the sharp bound on the first three consecutive higher order Schwarzian derivatives for Janowski convex functions. In fact, Theorem 1 is a generalization of the result in [8] (p. 8 Theorem 1 ) due to Dorff and Szynal.

Theorem 1. Let $f \in \mathcal{K}[A, B]$. Then, the following implications hold:

1. If $-1 \leq B<A \leq 1$, then $\left|\mathbf{S}_{3}\right| \leq(A-B)$.

2. (a) If either of the set of conditions:

$$
|3 A+B| \leq 1
$$

or $1 \leq|3 A+B| \leq 4$ and:

$$
\frac{4}{27}\left(\left|\frac{3 A+B}{2}\right|+1\right)^{3}-\left(\left|\frac{3 A+B}{2}\right|+1\right) \leq \frac{A(A+B)}{2} \leq 1
$$

hold, then $\left|\mathbf{S}_{4}\right| \leq 2(A-B)$.

(b) If $1 \leq|3 A+B| \leq 4$ and:

$$
-\frac{2}{3}\left(\left|\frac{3 A+B}{2}\right|+1\right) \leq \frac{A(A+B)}{2} \leq \frac{4}{27}\left(\left|\frac{3 A+B}{2}\right|+1\right)^{3}-\left(\left|\frac{3 A+B}{2}\right|+1\right)
$$

hold, then:

$$
\left|\mathbf{S}_{4}\right| \leq \frac{4(A-B)}{3}\left(\left|\frac{3 A+B}{2}\right|+1\right)\left(\frac{\left|\frac{3 A+B}{2}\right|+1}{3\left(\left|\frac{3 A+B}{2}\right|+\frac{A(A+B)}{2}+1\right)}\right)^{1 / 2} .
$$

3. If $-1<B<A<1$ and:

$$
\begin{aligned}
\left(A^{2}-2 A+2\right)(A-1)^{2}(A-B)^{2}(A+B-2)(A+B+2) & \\
& -36\left(A^{2}-1\right)^{2}(A+B-2)(A+B)+24(A+1)(A-1)^{2}(A-B) \geq 0
\end{aligned}
$$

hold, then $\left|\mathbf{S}_{5}\right| \leq 6(A-B)$. 
All estimates are sharp.

Proof. Let $f \in \mathcal{K}[A, B]$. For such a function $f$, by definition, we can write:

$$
1+\frac{z f^{\prime \prime}(z)}{f^{\prime}(z)}=\frac{1+A w(z)}{1+B w(z)}
$$

where $w(z)=c_{1} z+c_{2} z^{2}+c_{3} z^{3}+\cdots \in \mathcal{B}$. Comparing the coefficients of the like power terms in (8), we have:

$$
\begin{gathered}
a_{2}=\frac{1}{2}(A-B) c_{1}, \quad a_{3}=\frac{1}{6}(A-B)\left[(A-2 B) c_{1}^{2}+c_{2}\right], \\
a_{4}=\frac{1}{24}(A-B)\left[\left(A^{2}-5 A B+6 B^{2}\right) c_{1}^{3}+(3 A-7 B) c_{1} c_{2}+2 c_{3}\right]
\end{gathered}
$$

and:

$$
\begin{aligned}
a_{5}=\frac{1}{120}(A-B)\left[\left(A^{3}-24 B^{3}-9 A^{2} B+26 B^{2} A\right) c_{1}^{4}+\right. & 2\left(3 A^{2}-17 A B+23 B^{2}\right) c_{1}^{2} c_{2} \\
+ & \left.+3(A-3 B) c_{2}^{2}+4(2 A-5 B) c_{1} c_{3}+6 c_{4}\right]
\end{aligned}
$$

(1) From (9), we have:

$$
\begin{aligned}
\mathbf{S}_{3} & =6\left(a_{3}-a_{2}^{2}\right) \\
& =(A-B)\left[c_{2}-\frac{A+B}{2} c_{1}^{2}\right] .
\end{aligned}
$$

Now, an application of Lemma 2 in (12) gives the desired estimate on $\left|\mathbf{S}_{3}\right|$. The function for which equality holds is given by (8) with the choice $w(z)=z^{2}$.

(2) Next, we consider:

$$
\begin{aligned}
\mathbf{S}_{4} & =24\left(a_{4}-3 a_{2} a_{3}+2 a_{2}^{3}\right) \\
& =2(A-B)\left[c_{3}+\mu c_{1} c_{2}+v c_{1}^{3}\right] \\
& =2(A-B) Y(\mu, v),
\end{aligned}
$$

where $Y(\mu, v):=c_{3}+\mu c_{1} c_{2}+v c_{1}^{3}$ with $\mu:=-(3 A+B) / 2$ and $v:=A(A+B) / 2$.

Assume that $\Omega_{i}$ 's are as defined in Lemma 1 with $\mu$ and $v$ as given above. We observe that $v=A(A+B) / 2 \geq-1$ as $A B \geq-2-A^{2}$, and so, $(\mu, v) \notin \Omega_{3}$. Furthermore, $|\mu|=|-(3 A+B) / 2|<2$ because $-4<3 A+B<4$. Therefore, we can conclude that $(\mu, v) \notin \Omega_{i}(i=6,7,9,10,11,12)$. Moreover, $v<1$ as $A^{2}+A B<2$. This reveals that $(\mu, v) \notin \Omega_{5}$. We now claim that $(\mu, v) \notin \Omega_{4}$. For this, we first assume that $\mu \leq 0$. Then, $|\mu| \geq 1 / 2$ gives $3 A+B \geq 1$. Furthermore, the condition $v \leq(-2 / 3)(|\mu|+1)$ holds if $3 A(A+B) / 2 \leq-2(3 A+B+2)$ or equivalently if $-3 A(A+B) \geq$ $2(3 A+B+2)=2(3 A+B)+4 \geq 6$, that is if $-A B \geq 2+A^{2}$. Clearly, this is false. Similarly, in the case when $\mu \leq 0$, the condition $v \leq(-2 / 3)(|\mu|+1)$ does not hold. Thus, we conclude that our claim is true. Further, if $|3 A+B| \leq 1$, then $(\mu, v) \in \Omega_{1}$. Furthermore, if $1 \leq|3 A+B| \leq 4$ and:

$$
\frac{4}{27}\left(\left|-\frac{3 A+B}{2}\right|+1\right)^{3}-\left(\left|-\frac{3 A+B}{2}\right|+1\right) \leq \frac{A(A+B)}{2} \leq 1,
$$

then $(\mu, v) \in \Omega_{2}$. In view of Lemma 1 , we see that if $(\mu, v) \in \Omega_{1} \cup \Omega_{2}$, then $|\mathrm{Y}(\mu, v)| \leq 1$, and hence, $\left|\mathbf{S}_{4}\right| \leq 2(A-B)$. The function for which equality holds is given by (8) with the choice $w(z)=z^{3}$. 
Now, if $1 \leq|3 A+B| \leq 4$ and:

$$
-\frac{2}{3}\left(\left|\frac{3 A+B}{2}\right|+1\right) \leq \frac{A(A+B)}{2} \leq \frac{4}{27}\left(\left|\frac{3 A+B}{2}\right|+1\right)^{3}-\left(\left|\frac{3 A+B}{2}\right|+1\right),
$$

then $(\mu, v) \in \Omega_{8}$. Now, an application of Lemma 1, in this case, gives:

$$
\begin{aligned}
|\mathrm{Y}(\mu, v)| & \leq \frac{2}{3}(|\mu|+1)\left(\frac{|\mu|+1}{3(|\mu|+v+1)}\right)^{1 / 2} \\
& =\frac{2}{3}\left(\left|\frac{3 A+B}{2}\right|+1\right)\left(\frac{\left|\frac{3 A+B}{2}\right|+1}{3\left(\left|\frac{3 A+B}{2}\right|+\frac{A(A+B)}{2}+1\right)}\right)^{1 / 2} .
\end{aligned}
$$

This inequality together with (13) gives the desired bound on $\left|\mathbf{S}_{4}\right|$. To show the sharpness, we consider the function $f$ defined by (8) with the choice of the Schwarz function:

$$
w(z)=\frac{z\left(t_{1}-z\right)}{1-t_{1} z}
$$

where:

$$
t_{1}:=\left(\frac{\left|\frac{3 A+B}{2}\right|+1}{3\left(\left|\frac{3 A+B}{2}\right|+\frac{A(A+B)}{2}+1\right)}\right)^{1 / 2} .
$$

For this Schwarz function $w$, we see that $c_{1}=t_{1}, c_{2}=t_{1}^{2}-1, c_{3}=t_{1}^{3}-t_{1}$ and:

$$
\begin{aligned}
\mathbf{S}_{4} & =2(A-B)\left[c_{3}+\left|\frac{3 A+B}{2}\right| c_{1} c_{2}+\frac{A(A+B)}{2} c_{1}^{3}\right] \\
& =2(A-B)\left[t_{1}^{3}-t_{1}+\left|\frac{3 A+B}{2}\right| t_{1}\left(t_{1}^{2}-1\right)+\frac{A(A+B)}{2} t_{1}^{3}\right] \\
& =2(A-B)\left[t_{1}^{3}\left(\left|\frac{3 A+B}{2}\right|+\frac{A(A+B)}{2}+1\right)-t_{1}\left(\left|\frac{3 A+B}{2}\right|+1\right)\right] \\
& =\frac{4(A-B)}{3}\left(\left|\frac{3 A+B}{2}\right|+1\right)\left(\frac{\left|\frac{3 A+B}{2}\right|+1}{3\left(\left|\frac{3 A+B}{2}\right|+\frac{A(A+B)}{2}+1\right)}\right)^{1 / 2} .
\end{aligned}
$$

This confirms the sharpness of the result.

(3) Now, it remains to find the estimate on $\left|\mathbf{S}_{5}\right|$. Using (5)-(11), we get:

$$
\begin{aligned}
\mathbf{S}_{5} & =24\left(5 a_{5}-20 a_{2} a_{4}-9 a_{3}^{2}+48 a_{2}^{2} a_{3}-24 a_{2}^{4}\right) \\
& =3(A-B)\left[\hat{\gamma} p_{1}^{4}+\hat{a} p_{2}^{2}+2 \hat{\alpha} p_{1} p_{3}-(3 / 2) \hat{\beta} p_{1}^{2} p_{2}-p_{4}\right] \\
& =3(A-B) \Psi(\hat{\gamma}, \hat{a}, \hat{\alpha}, \hat{\beta}),
\end{aligned}
$$

where $\Psi(\hat{\gamma}, \hat{a}, \hat{\alpha}, \hat{\beta}):=\hat{\gamma} p_{1}^{4}+\hat{a} p_{2}^{2}+2 \hat{\alpha} p_{1} p_{3}-(3 / 2) \hat{\beta} p_{1}^{2} p_{2}-p_{4}$ with the parameters $\hat{\gamma}, \hat{a}, \hat{\alpha}$ and $\hat{\beta}$ given by:

$$
\hat{\gamma}:=\frac{(A-1)^{2}(2-A-B)}{16}, \hat{a}:=\frac{2-A-B}{4}, \hat{\alpha}:=\frac{1-A}{2}, \hat{\beta}:=\frac{(1-A)(3-B-2 A)}{6} .
$$

Since the case $A=1, B=-1$ was considered by Dorff and Szynal [8], we assume that $A$ and $B$ are constrained as $-1<B<A<1$. Under these conditions, it is a simple matter to verify that $0<\hat{\alpha}<1$ and $0<\hat{a}<1$. Moreover, the condition (6) holds if and only if: 


$$
\begin{aligned}
\frac{(A-1)^{2}\left(A^{2}-2 A+2\right)(A-B)^{2}(A+B-2)(A+B+2)}{1152}+ & \frac{1}{48}(A-1)^{2}(A+1)(A-B) \\
& \geq \frac{1}{32}\left(A^{2}-1\right)^{2}(A+B-2)(A+B)
\end{aligned}
$$

or equivalently, if and only if (7) holds. Therefore, in view of Lemma 3, we conclude that if the above condition holds, then we must have $|\Psi(\hat{\gamma}, \hat{a}, \hat{\alpha}, \hat{\beta})| \leq 2$, and thus, from (14), the result follows at once. Equality holds in the case of the function $f$ defined by (8) with the choice of the Schwarz function $w(z)=z^{4}$.

Remark 1. In particular, when $A=1$ and $B=-1$, Theorem 1 reduces to the result in [8] ( $p .8$ Theorem 1 ) due to Dorff and Szynal.

Example 1. Setting $A=1 / 2$ and $B=-1$ in Equation (3), we get the function $f_{1} \in \mathcal{K}$ defined by:

$$
f_{1}(z)=\frac{2(1-\sqrt{1-z})}{\sqrt{1-z}}=z+\frac{3 z^{2}}{4}+\frac{5 z^{3}}{8}+\frac{35 z^{4}}{64}+\frac{63 z^{5}}{128}+\cdots .
$$

Here, we see that, $B=-1<1 / 2=A, a_{2}=3 / 4, a_{3}=5 / 8, a_{4}=35 / 64$ and $\left|\mathbf{S}_{3}\right|=6\left|a_{3}-a_{2}^{2}\right|=3 / 8<$ $3 / 2=A-B$. This supports Part (1) of Theorem 1. Now, for the function $f_{1}$, we see that $3 A+B=1 / 2<1$ and $\left|\mathbf{S}_{4}\right|=24\left|a_{4}-3 a_{2} a_{3}+2 a_{2}^{3}\right|=3 / 8<2(A-B)=3$. This supports Part 2(a) of Theorem 1.

Example 2. Let $A=1 / 2$ and $B=-1$ in Equation (3). Then, we get the function $f_{2} \in \mathcal{K}$ defined by:

$$
f_{2}(z)=e^{z}-1=z+\frac{z^{2}}{2}+\frac{z^{3}}{6}+\frac{z^{4}}{24}+\frac{z^{5}}{120}+\cdots .
$$

Here, $1<|3 A+B|=3<4, a_{2}=1 / 2, a_{3}=1 / 6, a_{4}=1 / 24$ and $\left|\mathbf{S}_{4}\right|=24\left|a_{4}-3 a_{2} a_{3}+2 a_{2}^{3}\right|=1<$ $2(A-B)=2$. This supports Part 2(b) of Theorem 1. For an example satisfying Part (3) of Theorem 1, we set $A=3 / 4$ and $B=1 / 4$ in Equation (3), and thus, we get the function $f_{3}$ defined by:

$$
f_{3}(z)=z+\frac{z^{2}}{4}+\frac{z^{3}}{48}
$$

Here, $a_{2}=1 / 4, a_{3}=1 / 48, a_{4}=0, a_{5}=0$ and $\left|\mathbf{S}_{5}\right|=24\left|5 a_{5}-20 a_{2} a_{4}-9 a_{3}^{2}+48 a_{2}^{2} a_{3}-24 a_{2}^{4}\right|=27 / 32<$ $3=6(A-B)$.

Theorem 2. Let $f \in \mathcal{S}^{*}[A, B]$. Then, the following inequalities hold:

1. If $-1 \leq B<A \leq 1$, then $\left|\mathbf{S}_{3}\right| \leq 3(A-B)$.

2. (a) If $A$ and $B$ satisfy either:

$$
|B-3 A| \leq 1 / 2 \text { and }|A(2 A-B)| \leq 1
$$

or:

$$
1 / 2 \leq|B-3 A| \leq 1 \text { and } \frac{4}{27}(|B-3 A|+1)^{3}-(|B-3 A|+1) \leq A(2 A-B) \leq 1,
$$

then $\left|\mathbf{S}_{4}\right| \leq 8(A-B)$.

(b) Let us denote:

$$
T(A, B):=-\frac{2}{3}(|B-3 A|+1) .
$$


If either of the following sets of conditions:

$$
1 / 2 \leq|B-3 A| \leq 2, \quad T(A, B) \leq A(2 A-B) \leq \frac{4}{27}(|B-3 A|+1)^{3}-(|B-3 A|+1),
$$

or:

$$
|B-3 A| \geq 2, \quad T(A, B) \leq A(2 A-B) \leq \frac{2|B-3 A|(|B-3 A|+1)}{(B-3 A)^{2}+|B-3 A|+4}
$$

hold, then:

$$
\left|\mathbf{S}_{4}\right| \leq \frac{16(A-B)(|B-3 A|+1)^{3 / 2}}{3(3(|B-3 A|+A(2 A-B)+1))^{1 / 2}}
$$

(c) Moreover,

$$
\left|\mathbf{S}_{4}\right| \leq \begin{cases}8(A-B)|A(2 A-B)|, & 2 \leq|B-3 A| \leq 4, A(2 A-B) \geq\left((B-3 A)^{2}+8\right) / 12 ; \\ 48, & A=1, B=-1 .\end{cases}
$$

3. If $A$ and $B$ satisfy the conditions $0<(A+1)\left[43 A^{2}-43 A(B-1)+10 B^{2}-23 B+10\right]<80,0<$ $13 A-3 B+10<20$ and:

$$
\begin{gathered}
450(13 A-3 B-10)(13 A-3 B+10)\left(4 A^{2}-4 A B+B^{2}-1\right)^{2}-50(2 A-B-1)(2 A-B+1) \\
\left(8 A^{2}+A(7-9 B)+(B-7) B\right)^{2}-(13 A-3 B-10)(13 A-3 B+10)\left(1849 A^{4}-172 A^{3}(23 B-20)\right. \\
+A^{2}\left(2976 B^{2}-5916 B+2878\right)-4 A\left(230 B^{3}-798 B^{2}+928 B-231\right) \\
\left.+100 B^{4}-520 B^{3}+1177 B^{2}-630 B+98\right)(A-B)^{2} \leq 0, \quad(15)
\end{gathered}
$$

then $\left|\mathbf{S}_{5}\right| \leq 15(A-B)$.

All estimates are sharp.

Proof. Since $f \in \mathcal{S}^{*}[A, B]$, it follows that there exists a Schwarz function $w(z)=c_{1} z+c_{2} z^{2}+c_{3} z^{3}+\cdots$ such that:

$$
\frac{z f^{\prime}(z)}{f(z)}=\frac{1+A w(z)}{1+B w(z)} .
$$

Comparing the coefficients on both sides of (16), we have:

$$
\begin{gathered}
a_{2}=(A-B) c_{1}, \quad a_{3}=\frac{1}{2}(A-B)\left(A c_{1}^{2}-2 B c_{1}^{2}+c_{2}\right), \\
a_{4}=\frac{1}{6}(A-B)\left[(A-2 B)(A-3 B) c_{1}^{3}+(3 A-7 B) c_{1} c_{2}+2 c_{3}\right]
\end{gathered}
$$

and:

$$
\begin{aligned}
a_{5}=\frac{1}{24}(A-B)\left[\left(A^{3}-9 A^{2} B+26 A B^{2}-24 B^{3}\right) c_{1}^{4}+\right. & \left(6 A^{2}-34 A B+46 B^{2}\right) c_{1}^{2} c_{2} \\
& \left.+4(2 A-5 B) c_{1} c_{3}+3(A-3 B) c_{2}^{2}+6 c_{4}\right] .
\end{aligned}
$$

As in the proof of Theorem 1, using (17)-(19), we get:

$$
\mathbf{S}_{3}=-3(A-B)\left(A c_{1}^{2}-c_{2}\right), \mathbf{S}_{4}=8(A-B)\left[A(2 A-B) c_{1}^{3}+(B-3 A) c_{1} c_{2}+c_{3}\right]
$$


and:

$$
\begin{aligned}
\mathbf{S}_{5}=-\frac{3}{16}(A-B)\left[( A + 1 ) \left(43 A^{2}-43 A(B-1)+10 B^{2}-23 B\right.\right. & +10) p_{1}^{4} \\
-4\left(43 A^{2}+A(53-33 B)+5 B^{2}-23 B+15\right) p_{1}^{2} p_{2} & +80(2 A-B+1) p_{1} p_{3} \\
& \left.+4(13 A-3 B+10) p_{2}^{2}-80 p_{4}\right] .
\end{aligned}
$$

(1) From (20), we have:

$$
\begin{aligned}
\left|\mathbf{S}_{3}\right| & =3(A-B)\left|A c_{1}^{2}-c_{2}\right| \\
& \leq 3(A-B) \max \{1 ;|A|\} \\
& =3(A-B) .
\end{aligned}
$$

This gives the required estimate on $\left|\mathbf{S}_{3}\right|$. The extremal function in this case is given by (16) with the function $w(z)=z^{2}$.

(2) Again, from (20), we get:

$$
\begin{aligned}
\left|\mathbf{S}_{4}\right| & =8(A-B)\left|A(2 A-B) c_{1}^{3}+(B-3 A) c_{1} c_{2}+c_{3}\right| \\
& \leq 8(A-B)\left|c_{3}+\mu c_{1} c_{2}+v c_{1}^{3}\right|,
\end{aligned}
$$

where $\mu:=B-3 A$ and $v:=A(2 A-B)$. Assume that $\Omega_{i}^{\prime} s$ are as defined in Lemma 1 with the setting $\mu$ and $v$ mentioned above. In particular, $|\mu| \ngtr 4$ and $|\mu|=4$ if and only if $A=1$ and $B=-1$. It can be easily verified that for $A=1$ and $B=-1,(\mu, v) \notin \Omega_{11} \cup \Omega_{12}$. Now, in view of Lemma 1, from (22), we see that if $A$ and $B$ satisfy either:

$$
|B-3 A| \leq \frac{1}{2} \text { and }|A(2 A-B)| \leq 1
$$

or:

$$
\frac{1}{2} \leq|B-3 A| \leq 1 \text { and } \frac{4}{27}(|B-3 A|+1)^{3}-(|B-3 A|+1) \leq A(2 A-B) \leq 1,
$$

then $\left|\mathbf{S}_{4}\right| \leq 8(A-B)$. The extremal function in this case is given by (16) with the choice of the function $w(z)=z^{3}$.

Further, since $v>-1$, it follows that $(\mu, v) \notin \Omega_{3}$. Moreover, a computation reveals that $(\mu, v) \notin$ $\Omega_{4} \cup \Omega_{5}$. Furthermore, it can be verified that $(\mu, v) \in \Omega_{7}$ if and only if $A=1$ and $B=-1$. Now, an application of Lemma 1 gives:

$$
\left|\mathbf{S}_{4}\right| \leq \begin{cases}8(A-B)|A(2 A-B)|, & 2 \leq|B-3 A| \leq 4, A(2 A-B) \geq\left((B-3 A)^{2}+8\right) / 12 \\ 48, & A=1, B=-1 .\end{cases}
$$

The extremal function in this case is given by (16) with the choice of the function $w(z)=z$.

Similarly, we can prove that if either of the sets of following conditions:

$$
1 / 2 \leq|B-3 A| \leq 2,-\frac{2}{3}(|B-3 A|+1) \leq A(2 A-B) \leq \frac{4}{27}(|B-3 A|+1)^{3}-(|B-3 A|+1),
$$

or:

$$
|B-3 A| \geq 2,-\frac{2}{3}(|B-3 A|+1) \leq A(2 A-B) \leq \frac{2|B-3 A|(|B-3 A|+1)}{(B-3 A)^{2}+|B-3 A|+4}
$$

hold, then

$$
\left|\mathbf{S}_{4}\right| \leq \frac{16(A-B)(|B-3 A|+1)^{3 / 2}}{3(3(|B-3 A|+A(2 A-B)+1))^{1 / 2}}
$$


To show the sharpness, we consider the function $f$ defined by (16) with the choice of the Schwarz function $w(z)=z\left(t_{2}-z\right) /\left(1-t_{2} z\right)$, where:

$$
t_{2}:=\left(\frac{|B-3 A|+1}{3(|B-3 A|+A(2 A-B)+1)}\right)^{1 / 2} .
$$

For the Schwarz function $w$, given above, we see that $c_{1}=t_{2}, c_{2}=t_{2}^{2}-1, c_{3}=t_{2}^{3}-t_{2}$ and:

$$
\begin{aligned}
\mathbf{S}_{4} & =8(A-B)\left(A(2 A-B) c_{1}^{3}+|B-3 A| c_{1} c_{2}+c_{3}\right) \\
& =8(A-B)\left(A(2 A-B) t^{3}+|B-3 A| t_{2}\left(t_{2}^{2}-1\right)+t_{2}^{3}-t_{2}\right) \\
& =8(A-B)\left([A(2 A-B)+|B-3 A|+1] t_{2}^{3}-(|B-3 A|+1) t_{2}\right) \\
& =-\frac{16(A-B)}{3} \frac{(|B-3 A|+1)^{3 / 2}}{(3(|B-3 A|+A(2 A-B)+1))^{1 / 2}} .
\end{aligned}
$$

This confirms the sharpness of the result.

(3) Finally, it remains to find the estimate on $\left|\mathbf{S}_{5}\right|$. The expression for $\mathbf{S}_{5}$ given in (21) can be written as:

$$
\mathbf{S}_{5}=-\frac{15}{2}(A-B)\left[\hat{\gamma} p_{1}^{4}+\hat{a} p_{2}^{2}+2 \hat{\alpha} p_{1} p_{3}-(3 / 2) \hat{\beta} p_{1}^{2} p_{2}-p_{4}\right],
$$

where:

$$
\begin{gathered}
\hat{\gamma}:=(A+1)\left(43 A^{2}-43 A(B-1)+10 B^{2}-23 B+10\right) / 80, \hat{a}:=(13 A-3 B+10) / 20 \\
\hat{\alpha}:=(2 A-B+1) / 2, \quad \hat{\beta}:=\left(43 A^{2}+A(53-33 B)+5 B^{2}-23 B+15\right) / 30 .
\end{gathered}
$$

In order to apply Lemma 3, we assume that the parameters $A$ and $B$ satisfy the conditions $0<$ $(A+1)\left[43 A^{2}-43 A(B-1)+10 B^{2}-23 B+10\right]<80$ and $0<(13 A-3 B+10)<20$ together with the condition:

$$
\begin{gathered}
(13 A-3 B-10)(13 A-3 B+10)(A-B)^{2}\left(1849 A^{4}-172 A^{3}(23 B-20)\right. \\
+A^{2}\left(2976 B^{2}-5916 B+2878\right)-4 A\left(230 B^{3}-798 B^{2}+928 B-231\right) \\
\left.+100 B^{4}-520 B^{3}+1177 B^{2}-630 B+98\right) \\
+50(2 A-B-1)(2 A-B+1)\left(8 A^{2}+A(7-9 B)+(B-7) B\right)^{2} \\
\geq 450(13 A-3 B-10)(13 A-3 B+10)\left(4 A^{2}-4 A B+B^{2}-1\right)^{2},
\end{gathered}
$$

or equivalently, if (15) holds.

Thus, all conditions of Lemma 3 are fulfilled. Therefore, we have $\left|\mathbf{S}_{5}\right| \leq 15(A-B)$. The function $f$ defined by (16) with the choice of the Schwarz function $w(z)=z^{4}$ shows that the result is sharp. This completes the proof.

Example 3. Let $A=1 / 2$ and $B=-1$. Then, from Equation (2), we get the function $f_{4}$ defined by:

$$
f_{4}(z)=\frac{z}{(1-z)^{3 / 2}}=z+\frac{3 z^{2}}{2}+\frac{15 z^{3}}{8}+\frac{35 z^{4}}{16}+\frac{315 z^{5}}{128}+\frac{693 z^{6}}{256}+\cdots
$$

The function $f_{4} \in \mathcal{S}^{*}$ and satisfies the assertion in Part (1) of Theorem 2, as for this function, we have $a_{2}=3 / 2, a_{3}=15 / 8, a_{4}=35 / 16$ and $\left|\mathbf{S}_{3}\right|=6\left|a_{3}-a_{2}^{2}\right|=9 / 4<9 / 2=3(A-B)$. 
Example 4. (i) Let $A=1 / 2$ and $B=-1$ in Equation (2). Then, we get the function $f_{5} \in \mathcal{S}^{*}$ defined by:

$$
f_{5}(z)=z e^{z / 4}=z+\frac{z^{2}}{4}+\frac{z^{3}}{32}+\frac{z^{4}}{384}+\frac{z^{5}}{6144}+\cdots .
$$

Here, we have $|B-3 A|=3 / 4<1 / 2$ and $|A(2 A-B)|=1 / 8<1, a_{2}=1 / 4, a_{3}=1 / 32, a_{4}=1 / 384$ and $\left|\mathbf{S}_{4}\right|=1 / 4<2=8(A-B)$. This verifies the result asserted in Part 2(a) of Theorem 2.

(ii) Let $A=0$ and $B=-1$. Then, we have:

$$
T(A, B):=-\frac{2}{3}(|B-3 A|+1)=-\frac{4}{3} 1 / 2<|B-3 A|=1<2
$$

and

$$
T(A, B)=-\frac{4}{3}<A(2 A-B)=0<\frac{4}{27}(|B-3 A|+1)^{3}-(|B-3 A|+1)=12 .
$$

For $A=0$ and $B=-1$, we get the function $f_{6}$ from Equation (2) defined by:

$$
f_{6}(z)=\frac{z}{1-z}=z+z^{2}+z^{3}+z^{4}+z^{5}+\cdots .
$$

Now, a computation gives

$$
\left|\mathbf{S}_{4}\right|=0<\frac{32}{3 \sqrt{3}}=\frac{16(A-B)(|B-3 A|+1)^{3 / 2}}{3[3(|B-3 A|+A(2 A-B)+1)]^{1 / 2}} .
$$

This confirms the correctness of the assertion in 2(b) of Theorem 2.

(iii) For an example of a function satisfying the assertion in 2(c) of Theorem 2, we take $A=1$ and $B=-1$ in Equation (2). Then, we get the function $f_{7}$ defined by:

$$
f_{7}(z)=\frac{z}{(1-z)^{2}}=z+2 z^{2}+3 z^{3}+4 z^{4}+\cdots .
$$

For this function, it can be verified that $\left|S_{4}\right|=48$.

(iv) For $A=-1 / 4$ and $B=-1 / 2$, computations show that $0<13 A-3 B+10=33 / 4<20$ :

$$
0<(A+1)\left[43 A^{2}-43 A(B-1)+10 B^{2}-23 B+10\right]=\frac{507}{64}<80
$$

and Inequality (15) becomes $-2777538049 / 65536<0$. Setting $A=-1 / 4$ and $B=-1 / 2$ in (2), we get the function $f_{8}$ defined by:

$$
f_{8}(z)=z+\frac{z^{2}}{4}+\frac{5 z^{4}}{128}+\frac{35 z^{5}}{2048}+\cdots .
$$

For this function, we see that $\mathbf{S}_{\mathbf{4}}=0<15 / 4=15(A-B)$. This verifies the assertion in Part (3) of Theorem 2.

\section{Conclusion}

In Theorems 1 and 2, the sharp bounds on the first three consecutive derivatives for Janowski convex and star-like functions are investigated. Examples 1 and 2 support the conclusions of Theorem 1 , whereas Examples 3 and 4 validate the assertions in Theorem 2. The results obtained in this paper generalize several existing results in this direction, and they are pointed out. It would be interesting to investigate the estimation on other higher order Schwarzian derivatives and their applications to study the properties of a non-linear dynamical system.

Author Contributions: All authors contributed equally.

Acknowledgments: The authors would like to express their gratitude to the referees for many valuable suggestions regarding a previous version of this paper.

Funding: The first author was supported by the Basic Science Research Program through the National Research Foundation of Korea (NRF) funded by the Ministry of Education, Science and Technology (No. 2016R1D1A1A09916450) 
Conflicts of Interest: The authors declare no conflict of interest.

\section{References}

1. Janowski, W. Extremal problems for a family of functions with positive real part and for some related families. Ann. Polon. Math. 1970/1971, 23, 159-177. [CrossRef]

2. Nehari, Z. The Schwarzian derivative and schlicht functions. Bull. Am. Math. Soc. 1949, 55, 545-551. [CrossRef]

3. Kraus, W. Über den Zusammenhang einiger Characteristiken eines einfach zusammenhängenden Bereiches mit der Kreisabbildung. Mitt. Math. Sem. Giessen. 1932, 21, 1-28.

4. Hille, E. Remarks on a paper by Zeev Nehari. Bull. Amer. Math. Soc. 1949, 55, 552-553. [CrossRef]

5. Nehari, Z. A property of convex conformal maps. J. Anal. Math. 1976, 30, 390-393. [CrossRef]

6. Harmelin, R. Aharonov invariants and univalent functions. Israel J. Math. 1982, 43, 244-254. [CrossRef]

7. Schippers, E. Distortion theorems for higher order Schwarzian derivatives of univalent functions. Proc. Am. Math. Soc. 2000, 128, 3241-3249. [CrossRef]

8. Dorff, M.; Szynal, J. Higher order Schwarzian derivatives for convex univalent functions. Tr. Petrozavodsk. Gos. Univ. Ser. Mat. 2009, 15, 7-11.

9. Koepf, W. Close-to-convex functions and linear-invariant families. Ann. Acad. Sci. Fenn. Ser. A I Math. 1983, 8, 349-355. [CrossRef]

10. Gal, S.G. Higher order derivatives of Schwarz, Sălăgean and Rushcheweyh types in the geometric theory of complex functions. Rev. Roumaine Math. Pures Appl. 2002, 47, 33-42.

11. Miller, S.S.; Mocanu, P.T. Second order differential inequalities in the complex plane. J. Math. Anal. Appl. 1978, 65, 289-305. [CrossRef]

12. Tamanoi, H. Higher Schwarzian operators and combinatorics of the Schwarzian derivative. Math. Ann. 1996, 305, 127-151. [CrossRef]

13. Kim, S.A.; Sugawa, T. Invariant Schwarzian derivatives of higher order. Complex Anal. Oper. Theory 2011, 5, 659-670. [CrossRef]

14. Aharonov, D. A necessary and sufficient condition for univalence of a meromorphic function. Duke Math. J. $1969,36,599-604$.

15. Kim, S.A.; Sugawa, T. Invariant differential operators associated with a conformal metric. Michigan Math. J. 2007, 55, 459-479.

16. Schippers, E. The calculus of conformal metrics. Ann. Acad. Sci. Fenn. Math. 2007, 32, 497-521

17. Hacibekirog̃lu, G.; Çãğlar, M.; Polatog̃lu, Y. The higher order Schwarzian derivative: Its applications for chaotic behavior and new invariant sufficient condition of chaos. Nonlinear Anal. Real World Appl. 2009, 10, 1270-1275. [CrossRef]

18. Aguilar, R.M. Higher order Schwarzians for geodesic flows, moment sequences and the radius of adapted complexifications. Q. J. Math. 2013, 64, 1-36. [CrossRef]

19. Kwon, O.; Sim, Y. Starlikeness and Schwarzian derivatives of higher order of analytic functions. Commun. Korean Math. Soc. 2017, 32, 93-106. [CrossRef]

20. Prokhorov, D.V.; Szynal, J. Inverse coefficients for $(\alpha, \beta)$-convex functions. Ann. Univ. Mariae Curie-Skłodowska Sect. A 1981, 35, 125-143.

21. Keogh, F.R.; Merkes, E.P. A coefficient inequality for certain classes of analytic functions. Proc. Am. Math. Soc. 1969, 20, 8-12. [CrossRef]

22. Ali, R.M.; Ravichandran, V.; Seenivasagan V. Coefficient bounds for p-valent functions. Appl. Math. Comput. 2007, 187, 35-46. [CrossRef]

23. Ravichandran, V.; Verma, S. Bound for the fifth coefficient of certain star-like functions. C. R. Math. Acad. Sci. Paris 2015, 353, 505-510. [CrossRef]

(C) 2018 by the authors. Licensee MDPI, Basel, Switzerland. This article is an open access article distributed under the terms and conditions of the Creative Commons Attribution (CC BY) license (http://creativecommons.org/licenses/by/4.0/). 